\title{
Mise en œuvre de l'intervention du Conseil fédéral - un défi tarifaire
}

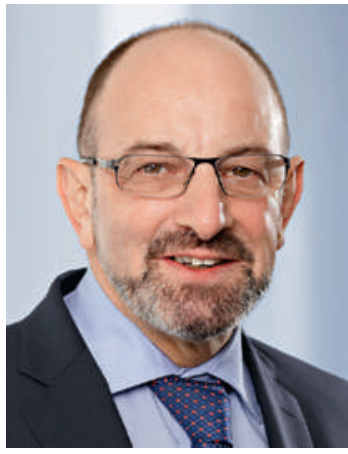

Cette fois, nous y sommes: l'ordonnance sur l'adaptation de structures tarifaires dans l'assurance-maladie du Conseil fédéral entrera en vigueur au $1^{\text {er }}$ octobre 2014. Comme prévu, la mise en œuvre de cette intervention isolée dans une structure tarifaire fondée sur des règles économiques précises n'a pas été chose simple. A maintes reprises, la FMH a signalé que les transferts linéaires dans la structure tarifaire étaient inadéquats et créeraient de nouveaux déséquilibres.

Suite à la publication de l'ordonnance définitive, les partenaires tarifaires ont immédiatement initialisé le développement d'une banque de données commune. Ils se sont mis relativement rapidement d'accord sur la plupart des points, mais la formulation du texte de l'ordonnance est malheureusement tout sauf précise, en particulier quand elle se contente de limiter la facturation de la position 00.0015 aux «prestations de la médecine de famille». Que sont au juste les prestations de la médecine de famille? Comment sont-elles définies? Ces questions ont, déjà par le passé, suscité d'interminables discussions au sein des organes de la FMH, sans aboutir à un résultat convaincant. Par courrier du 21 août 2014, l'Office fédéral de la santé publique (OFSP) a informé les partenaires tarifaires que la mise en œuvre technique et l'interprétation médicale revenaient aux partenaires, au nom de l'autonomie tarifaire. Dès lors, il s'agit de trouver des solutions partenariales, conformément au principe de la LAMal.

\section{Pour la FMH et MFE, il est hors de question de faire une distinction aussi radicale dans le TARMED entre les prestations de la médecine de famille et les autres prestations.}

La question de savoir comment différencier les prestations spécialisées de celles relevant de la médecine de famille - et donc pouvant être cumulées avec la position 00.0015 - se pose principalement pour les spécialistes en médecine interne générale porteurs d'un deuxième titre de spécialiste. Selon l'OFSP, c'est le principe de confiance qui prédomine dans la LAMal, autrement dit, la Confédération s'en remet à l'expertise et à la bonne foi des médecins. Dans sa réponse, l'OFSP ne précise cependant rien sur la manière de contrôler l'autorisation de facturer: par le contrôle des factures via les assureurs, par des restrictions de facturation, par des règles de cumulation ou encore par l'introduction d'une liste de personnes habilitées à facturer cette prestation dans la structure tarifaire? Pour leur part, les assureurs veulent distinguer clairement les prestations de la médecine de famille en intégrant un groupe de prestations dans la structure tarifaire. Ils s'assureraient ainsi un contrôle automatique des factures et minimiseraient nettement les risques de leur côté.

Selon le département Tarifs et conventions pour la médecine ambulatoire en Suisse et Médecins de famille Suisse (MFE), il est hors de question à l'heure actuelle de faire une distinction aussi radicale dans le TARMED entre les prestations de la médecine de famille et les autres prestations. Le Bureau des tarifs (représentants des organisations faîtières) tout comme le Comité central et l'Assemblée des délégués de

\section{Les nouvelles positions sont tarifées et peuvent être facturées dès le 1 er octobre.}

la FMH appuient la décision stratégique de développer une banque de données commune sans restriction ou, à la rigueur, d'y intégrer une liste négative réduite en vue de créer des garde-fous pour les porteurs de deux titres et de permettre de ne réglementer que là où c'est vraiment justifié.

Malgré d'intenses négociations, au 15 septembre 2014, les assureurs (curafutura, CTM et santésuisse) ne s'étaient pas laissé convaincre par cette liste négative. C'est donc sans l'accord de la FMH qu'ils ont intégré un groupe de prestations dans leurs propres systèmes de validation - c'est-à-dire à l'extérieur de la structure tarifaire - afin notamment de pouvoir automatiser le contrôle des factures. En revanche, tous les partenaires tarifaires se sont mis d'accord sur la conception du navigateur tarifaire et sur un monitorage commun afin de pouvoir réagir rapidement en cas de développements indésirables.

Un point est garanti, les nouvelles positions sont tarifées et peuvent donc être facturées dès le $1^{\text {er }}$ octobre. Sur le plan juridique, les recours déposés par la fmCh et $\mathrm{H}+$ ainsi que par différents hôpitaux sont encore pendants. Le Tribunal administratif fédéral s'est déclaré compétent en la matière et a demandé au Conseil fédéral de prendre position dans un délai d'un mois, d'ici fin septembre. En d'autres termes, aucune décision ne sera prise avant l'entrée en vigueur de l'ordonnance et aucun effet suspensif n'est donc attendu. Le nouveau tarif entrera dès lors en vigueur au $1^{\mathrm{er}}$ octobre 2014.

Dr Ernst Gähler, vice-président de la FMH, responsable du département Tarifs et conventions pour la médecine ambulatoire en Suisse 